\title{
复数的应用
}

\section{Application of Complex Number}

\section{张倩瑶 黄星}

\author{
Qianyao Zhang Xing Huang
}

\section{南阳职业学院}

中国・河南 南阳 474500

Nanyang Vocational College,

Nanyang, Henan, 474500, China
【摘要】在给南阳职业学院学生讲授高等数学课的过程中, 发现学生的高中基础比较差, 有必要对中学的数学基础进行复习巩固。如何在短时间内对中学数学进行复习, 有利于学 生更好地学习高等数学呢? 这就对备课、讲课提出了很高的要求。通过研究发现, 运用复数 的特性把一些数学知识点串联起来, 可提高课堂效率。

【Abstract】In the process of teaching higher mathematics to the students of Nanyang Vocational College, it is found that the high school foundation of the students is relatively poor, so it is necessary to review and consolidate the mathematics foundation of the middle school. How to carry on the review to the middle school mathematics in a short time, is beneficial to the student to learn the higher mathematics better? This puts forward high requirements for the preparation of lessons and lectures. Through the research, people find that by using the characteristics of complex number, some mathematical knowledge points are connected in series, which can improve the classroom efficiency in practical teaching.

【关键词】复数; 性质; 应用

【Keywords】complex number; nature; application

【DOI】10.36012/sde.v1i3.507

\section{1 复数的概念、表示法及其性质}

\section{1 复数的概念}

人们把形如 $z=a+b i$ ( $a, b$ 均为实数 $)$ 的数称为复数, 其中 $a$ 称为实部, $b$ 称为虚部, $i$ 称为虚数单位。当虚部等于零时, 这 个复数可视为实数; 当虚部不等于零时, 实部等于零, 常称 $z$ 为纯虚数。

\section{2 复数的几种表示法}

(1)复数 $z$ 的代数形式为: $z=a+b i$;2复数 $z$ 的三角形式为: $z=|z||\cos \theta+i| z \mid \sin \theta\left(|z|=\sqrt{\left(a^{2}+b^{2}\right)}, \theta=\arctan \frac{b}{a}\right)$; (3)复数 $z$ 的 指数形式为: $z=|z| e^{i \theta}\left(e^{i \theta}=\cos \theta+i \sin \theta\right)$; (4) 复数 $z$ 的极坐标形式 为: $z=|z| \angle \theta$; (5)复数 $z$ 的代数形式和极坐标形式之间的转换 公式: $a=|z| \cos \theta, b=|z| \sin \theta,|z|=\sqrt{\left(a^{2}+b^{2}\right)}, \theta=\arctan \frac{b}{a}$; (6)特 别量: $1=1 \angle 0^{\circ},-1=1 \angle 180^{\circ}, i=1 \angle 90^{\circ},-i=1 \angle-90^{\circ}$; (7)几何形
式:复平面上的点 $z(a, b)$ 或由原点出发的向量 $\overrightarrow{O z}$ 。X 轴叫作 实轴, $Y$ 轴口丩作虚轴, 虚轴上的点除原点外都表示纯虚数, 各 象限内的点表示虚部不为零的虚数 (因为原点所对应的有序 实数对为 $(0,0)$, 表示实数)。

\section{3 复数的性质}

(1)复数不能比较大小; (2)复数相等 $a+b i=c+d i \Leftrightarrow a=c, b=d$ ( $a, b, c, d$ 都是实数); (3)复数模的几何意义:代表复数 $z=a+b i$ 在复平面上对应的点 $z(a, b)$ 到原点的距离, 即 $|z|=|o z|=$ $\sqrt{\left(a^{2}+b^{2}\right)}$

\section{4 复数的加、减运算法则}

若 $z_{1}=a+b i, z_{2}=c+d i\left(a, b, c, d\right.$ 都是实数), 加法法则: $z_{1}+z_{2}=$ $(a+c)+(b+d) i$; 减法法则: $z_{1}-z_{2}=(a-c)+(b-d) i$ 。

\section{5 复数加法运算的几何意义}

若有 $z_{1}(a, b) 、 z_{2}(c, d) 、 z(a+c, b+d)$, 则 $z_{1}+z_{2}=\overrightarrow{o z_{1}}+\overrightarrow{o z_{2}}=$ $\overrightarrow{o z}$,符合向量加法的平行四边形法则。 


\section{2 复数的应用举例}

设 $z=\cos \theta+i \sin \theta$, 则 $\bar{z}=\cos \theta-i \sin \theta$ 且 $z \bar{z}=1, \frac{1}{z}=\bar{z}=\cos \theta-$ $i \sin \theta$, 易得 $\cos \theta=\frac{1}{2}\left(z+\frac{1}{z}\right)=\frac{z^{2}+1}{2 z} ; \sin \theta=\frac{1}{2 i}\left(z-\frac{1}{z}\right)=\frac{z^{2}-1}{2 z i}$ ； $\tan \theta=\frac{\sin \theta}{\cos \theta}=\frac{z^{2}-1}{\left(z^{2}+1\right) i}$

根据棣美弗定理, 可以得出:

$z^{\mathrm{n}}=(\cos \theta+i \sin \theta)^{\mathrm{n}}=\cos n \theta+i \sin n \theta ; \quad \frac{1}{z^{\mathrm{n}}}=(\cos \theta-i \sin \theta)^{\mathrm{n}}=\cos n \theta-$ $i \sin n \theta$

进而可得

$\cos n \theta=\frac{1}{2} \quad\left(z^{\mathrm{n}}+\frac{1}{z^{\mathrm{n}}}\right)=\frac{z^{2 \mathrm{n}}+1}{2 z^{\mathrm{n}}} ; \sin n \theta=\frac{1}{2 i} \quad\left(z^{\mathrm{n}}-\frac{1}{z^{\mathrm{n}}}\right)=\frac{z^{2 \mathrm{n}}-1}{2 z^{\mathrm{n} i}} ；$ $\tan n \theta=\frac{\sin \theta}{\cos \theta}=\frac{z^{2 n}-1}{\left(z^{2 n}+1\right) i}$

另外, 若 $z_{1}=\cos \theta_{1}+i \sin \theta_{1}, z_{2}=\cos \theta_{2}+i \sin \theta_{2}$

则 $z_{1} z_{2}=\cos \left(\theta_{1}+\theta_{2}\right)+i \sin \left(\theta_{1}+\theta_{2}\right), \overline{z_{z_{2}}}=\cos \left(\theta_{1}+\theta_{2}\right)-i \sin \left(\theta_{1}+\theta_{2}\right)$

易得:

$\cos \left(\theta_{1}+\theta_{2}\right)=\frac{1}{2}\left(z_{1} z_{2}+\frac{1}{z z_{2}}\right)=\frac{z_{1}^{2} z_{2}^{2}+1}{2 z z_{2}}$

$\sin \left(\theta_{1}+\theta_{2}\right)=\frac{1}{2 i}\left(z_{1} z_{2}-\frac{1}{z_{1} z_{2}}\right)=\frac{z_{1}^{2} z_{2}^{2}-1}{2 z_{1} z_{2} i}$

$\cos \left(\theta_{1}-\theta_{2}\right)=\frac{1}{2}\left(\frac{z_{1}}{z_{2}}+\frac{z_{2}}{z_{1}}\right)=\frac{z_{1}^{2}+z_{2}^{2}}{2 z_{1} z_{2}}$

$\sin \left(\theta_{1}-\theta_{2}\right)=\frac{1}{2 i}\left(\frac{z_{1}}{z_{2}}-\frac{z_{2}}{z_{1}}\right)=\frac{z_{1}^{2}-z_{2}^{2}}{2 z_{1} z_{2} i}$

由此可以看出, 三角函数都能用复数表示出来。因此, 利 用这些关系式将三角问题转化为数的问题, 然后借助代数知 识解答三角题应是一件很自然的事情哑。事实上,这些关系在 三角的求值、化简、恒等变形、解方程等中有广泛的应用。下面 举例说明:

例 1 求证: $\cos 3 \alpha=4(\cos \alpha)^{3}-3 \cos \alpha$

证明: 设 $z=\cos \alpha+i \sin \alpha$, 则

$4(\cos \alpha)^{3}-3 \cos \alpha=4\left[\frac{1}{2}\left(z+\frac{1}{z}\right)\right]^{3}-3 \frac{1}{2}\left(z+\frac{1}{z}\right)$

$=\frac{1}{2}\left(z^{3}+3 z+\frac{3}{z}+\frac{1}{z^{3}}\right)-\frac{3}{2}\left(z+\frac{1}{z}\right)=\frac{1}{2}\left(z^{3}+\frac{1}{z^{3}}\right)=\cos 3 \alpha$

\section{3 复数在向量、几何中的应用}

例 1 平面上圆的方程为 $A z \bar{z}+\bar{\beta} z+\beta \bar{z}+c=0$ （其中 $A, C$ 为实 数, $\beta$ 为复数), 证明: $A\left(x^{2}+y^{2}\right)+B x+D y+C=0$ ( $A, C$ 为实数 $)$ 是 圆的方程。
令 $x=\frac{z+\bar{z}}{2}, y=\frac{z-\bar{z}}{2 i}$

$x^{2}+y^{2}=\left(\frac{z+\bar{z}}{2}\right)^{2}+\left(\frac{z-\bar{z}}{2 i}\right)^{2}=z \bar{z}$

$A z \bar{z}+B \frac{z+z}{2}+D \frac{z-z}{2 i}+\mathrm{C}=0$

$A z \bar{z}+\left(\frac{B}{2}-\frac{D}{2} i\right)_{\mathrm{z}}+\left(\frac{B}{2}+\frac{D}{2} i\right)_{\bar{z}}+C=0$

令 $\beta=\frac{B}{2}+\frac{D}{2} i$, 则 $A z \bar{z}+\bar{\beta} z+\beta \bar{z}+c=0$

\section{4 复数在不等式中的应用}

本文将把复数的模的一些基本性质应用到实数不等式的 证明中, 对于一些较为复杂的不等式给出简单的证明。

记号说明:

$|z|$ 表示 $z$ 的模; $z$ 表示 $z$ 的共轭复数; 英文字母 $z, z_{1}, z_{2} \cdots$ 等 表示复数; $\operatorname{Re} z$ 表示 $z$ 的实部; $\operatorname{Im} z$ 表示 $z$ 的虚部。

首先将复数模的一些性质列举如下:

$|z|=\sqrt{|\operatorname{Re} z|^{2}+|\operatorname{Im} z|^{2}} ;\left|z_{1} z_{2}\right|=\left.\left|z_{1}\right| z_{2}|;| z\right|^{2}=|\bar{z}|^{2}=z \bar{z}$ 证明: 可以用复数指数表示

令 $z=\mathrm{re}^{\mathrm{i \theta} \theta},|z|^{2}=r^{2}$

$z \bar{z}=r e^{i \theta} r e^{-i \theta}=r^{2}$

(1) $|\operatorname{Re} z| \leqslant|z|,|\operatorname{Im} z| \leqslant|z| ;(2)\left|z_{1}+z_{2}\right| \leqslant\left|z_{1}\right|+\left|z_{2}\right|$;3) $\left|z_{1}-z_{2}\right|$ $\geqslant\left|z_{1}\right|-\left|z_{2}\right|$; (4) $\frac{|\operatorname{Re} z|+|\operatorname{Im} z|}{\sqrt{2}} \leqslant|z| \leqslant|\operatorname{Re} z|+|\operatorname{Im} z|$; (5) $|\operatorname{Re} z \operatorname{Im} z| \leqslant \frac{|z|^{2}}{\sqrt{2}}$ 。

证明: $(|\operatorname{Re} z|-|\operatorname{Im} z|)^{2}=|z|^{2}-2|\operatorname{Re} z||\operatorname{Im} z| \geqslant 0$

例 1 设 $a_{1} 、 a_{2} 、 b_{1} 、 b_{2}$ 为任意实数,试证:

$\left|a_{1} b_{1}+a_{2} b_{2}\right| \leqslant \sqrt{a_{1}^{2}+a_{2}^{2}} \sqrt{b_{1}{ }^{2}+b_{2}{ }^{2}}$

（该不等式证法有很多,用复数模的性质证,显得极为简单。）

证明: 令 $z_{1}=a_{1}+i a_{2}, z_{2}=b_{1}-i b_{2}$

则 $z_{3}=z_{1} z_{2}=\left(a_{1}+i a_{2}\right)\left(b_{1}-i b_{2}\right)$

由(2)、(3)得:

$\left|a_{1} b_{1}+a_{2} b_{2}\right|=\left|\operatorname{Re} z_{3}\right| \leqslant\left|z_{3}\right|=\left|z \overline{z_{2}}\right|=\left|z_{1}\right|\left|\bar{z}_{2}^{-}\right|=\sqrt{a_{1}^{2}+a_{2}{ }^{2}}$ $\sqrt{b_{1}{ }^{2}+b_{2}{ }^{2}}$

参考文献

[1]彭翕成,张景中.点几何的解题应用:复数恒等式篇 $[\mathrm{J}]$.数学通 报,2019,58(5):1-4. 